\title{
Admissible Equilibria of Non-neutral Plasmas in a Malmberg-Penning Trap
}

\author{
Igor Kotelnikov \\ Budker Institute of Nuclear Physics, Lavrentyev Avenue 11, Novosibirsk, 630090, Russia \\ Massimiliano Romé \\ I.N.F.N. Sezione di Milano and Dipartimento di Fisica, Università degli Studi di Milano, Via Celoria 16, I-20133 Milano, Italy
} (Received 5 May 2008; published 22 August 2008)

\begin{abstract}
A "parallel current constraint" is derived, that in combination with the Poisson equation allows one to select admissible equilibria of non-neutral plasmas in a Malmberg-Penning trap in the presence of a nonuniform and nonaxisymmetric magnetic field. Asymmetry-induced currents (analogous to the PfirschSchlüter currents in Tokamaks) appearing in a non-neutral plasma even in the absence of magnetic drifts are explicitly computed in the case of a uniformly tilted magnetic field.
\end{abstract}

DOI: 10.1103/PhysRevLett.101.085006

PACS numbers: 52.27.Jt, 52.27.Aj

The radial confinement of non-neutral plasmas in Malmberg-Penning (MP) traps is provided by a strong axial magnetic field. This field is assumed to be uniform in most theories that deal with plasma confinement. However, small perturbations of the magnetic field may play a crucial role in the transport of non-neutral plasmas in this kind of confinement devices [1]; see also the review paper [2] and references therein for further discussion of the problem of non-neutral plasma transport. On the other hand, it is well known that an accurate treatment of the plasma transport requires at first an analysis of the plasma equilibrium, as it is demonstrated by established theories for quasineutral plasma confined, e.g., in tandem mirrors [3].

It may be wondered whether an equilibrium of a nonneutral plasma exists in an asymmetric magnetic field, since an asymmetry leads in general to plasma expansion. A positive answer to this question implies that the equilibrium is referred to a time interval shorter than the expansion time $\tau_{m}$. If the asymmetry is small, the latter is expected to be at least greater then the axial bounce time of the particles inside the trap $\tau_{b}$ and the plasma azimuthal rotation time $2 \pi / \omega_{E}$, i.e., $\tau_{m} \gg\left(\tau_{b}, 2 \pi / \omega_{E}\right)$. In general, $\tau_{m} \propto \epsilon^{-2}$, where the parameter $\epsilon$ characterizes the smallness on the magnetic field inhomogeneity. At this stage it can be assumed that $\epsilon \sim \delta B / B$, where $\delta \mathbf{B}$ represents the difference of the actual magnetic field from an ideal uniform magnetic field $\mathbf{B}_{*}=B_{*} \mathbf{e}_{z}$ directed along the symmetry axis of the cylindrical confinement device. For the small $\epsilon$ values achieved in existing devices the expansion time can therefore be quite large, and for a shorter time interval, $t \ll \tau_{m}$, it is possible to consider a slowly evolving plasma column as being in a static equilibrium.

Systematic studies of nonaxisymmetric equilibria in a MP trap have been started in Refs. [4,5]. In Ref. [4] the equilibrium of a non-neutral plasma column in a weakly tilted magnetic field was simulated numerically. In Ref. [5] an electrostatic asymmetry was introduced by azimuthally sectored electrodes, and the analytical treatment was limited to the case of a cold plasma with a stepwise radial density profile. Later on, three-dimensional numerical particle-in-cell simulations of the non-neutral plasma equilibrium with quadrupole or mirror magnetic perturbations have been reported in Ref. [6]. However, similar numerical simulations are hardly able to uncover fine-structure effects that limit plasma lifetime in existing and future facilities designed to achieve improved confinement of non-neutral plasmas.

In Ref. [7] the equilibrium of non-neutral plasmas on a set of nested toroidal magnetic surfaces has been recently considered. This work together with the theory of quasineutral plasma equilibria in tandem mirrors [3] bestows a guideline of how to establish a constraint on the shape of admissible plasma equilibria. Together with Poisson's equation, rewritten in flux coordinates, this constraint constitutes a self-consistent method for determining asymmetric equilibria of non-neutral plasmas in a MP trap.

The approach is based on the use of curvilinear flux coordinates for the magnetic field. As it was argued in Ref. [8], performing the calculations in flux coordinates makes the interpretation of the plasma equilibrium much easier and provides the best approach to the problem of the error field mediated transport.

The electric current produced by the flowing electrons confined in a MP trap produces a negligible change of the magnetic field, if the electron density $n$ is far below the Brillouin limit [9] $n_{B} \equiv B^{2} / 8 \pi m c^{2}$ (with $m$ the particle mass and $c$ the speed of light) except for the case of a fast rotating non-neutral plasma equilibrium [10]. The magnetic field can then be described by a scalar magnetic potential $\chi$ such that

$$
\mathbf{B}=\nabla \chi
$$

Alternatively, any divergence-free field can be written as

$$
\mathbf{B}=\nabla \psi \times \nabla \vartheta
$$


where $\psi$ and $\vartheta$ are flux coordinates [11], which are constant along the magnetic field lines. The relations $\psi=$ $\psi(x, y, z), \vartheta=\vartheta(x, y, z), \chi=\chi(x, y, z)$ define a system of curvilinear coordinates.

The momentum balance equation for a pure electron plasma is

$$
m n\left(\frac{\partial \mathbf{v}}{\partial t}+\mathbf{v} \cdot \boldsymbol{\nabla} \mathbf{v}\right)=e n\left(\frac{1}{c} \mathbf{v} \times \mathbf{B}-\boldsymbol{\nabla} \phi\right)-\boldsymbol{\nabla} p,
$$

where $e$ is the particle charge, $\mathbf{v}$ the fluid velocity, $\phi$ the electrostatic potential, and $p$ the scalar pressure. In the equilibrium state, the time derivative vanishes. If the electron density is far below the Brillouin limit, $n \ll n_{B}$, and the plasma column is in a slow rotation state [10], then the $\mathbf{v} \cdot \boldsymbol{\nabla} \mathbf{v}$ term is negligible in comparison with the other terms and the force balance equation reduces to

$$
\boldsymbol{\nabla} p=e n\left(\frac{1}{c} \mathbf{v} \times \mathbf{B}-\boldsymbol{\nabla} \phi\right) .
$$

Dotting Eq. (3) with $\mathbf{B}$, one finds that $\mathbf{B} \cdot \boldsymbol{\nabla} p=-e n \mathbf{B} \cdot$ $\nabla \phi$. The electron temperature $T$ tends to be constant along the magnetic field, $\mathbf{B} \cdot \boldsymbol{\nabla} T=0$. When this situation is reached, the electron density must have the form

$$
n=N(\psi, \vartheta) \exp \left[-\frac{e \phi}{T(\psi, \vartheta)}\right]
$$

and must also be consistent with the Poisson equation. Therefore, the fundamental equilibrium equation for a pure electron plasma is

$$
\nabla^{2} \phi(\psi, \vartheta, \chi)=-4 \pi e N(\psi, \vartheta) \exp \left[-\frac{e \phi}{T(\psi, \vartheta)}\right] .
$$

This equation contains two functions of $\psi$ and $\vartheta, N(\psi, \vartheta)$ and $T(\psi, \vartheta)$ which are subject to a constraint derived below. The dependence of $N$ and $T$ on $\vartheta$ makes the plasma equilibria in MP traps very different from those obtained in toroidal devices [7], where the functions $N(\psi)$ and $T(\psi)$ are entirely determined by the experimental conditions and by the plasma transport processes.

The equilibrium equation (3) implies

$$
\mathbf{v}=\frac{v_{\|}}{B} \mathbf{B}-c\left(\frac{\nabla p}{e n}+\nabla \phi\right) \times \frac{\mathbf{B}}{B^{2}},
$$

leaving undefined the velocity $\boldsymbol{v}_{\|}$parallel to the magnetic field. According to Eq. (4), the pressure

$$
p(\psi, \vartheta, \phi)=T(\psi, \vartheta) N(\psi, \vartheta) \exp [-e \phi / T(\psi, \vartheta)],
$$

is a function of $\psi, \vartheta$, and $\phi$. Thus,

$$
\boldsymbol{\nabla} p=\frac{\partial p}{\partial \psi} \boldsymbol{\nabla} \psi+\frac{\partial p}{\partial \vartheta} \boldsymbol{\nabla} \vartheta-e n \boldsymbol{\nabla} \phi .
$$

Combining Eqs. (6) and (8), the $\boldsymbol{\nabla} \phi$ terms cancel, and

$$
\mathbf{v}=v_{\|} \frac{\mathbf{B}}{B}-c \frac{\partial p}{\partial \psi} \frac{\boldsymbol{\nabla} \psi \times \mathbf{B}}{e n B^{2}}-c \frac{\partial p}{\partial \vartheta} \frac{\boldsymbol{\nabla} \vartheta \times \mathbf{B}}{e n B^{2}} .
$$

This relation reveals that $\partial p / \partial \psi$ and $\partial p / \partial \vartheta$ cannot be neglected even when $p$ vanishes in the zero temperature limit, as explained in Ref. [7].

The parallel component $v_{\|}$of the plasma flow, Eq. (9), must be consistent with the steady-state constraint

$$
\boldsymbol{\nabla} \cdot(e n \mathbf{v})=0 .
$$

This constraint leaves a net parallel electric current of the non-neutral plasma undetermined in a toroidal confinement configuration [7], but it leads to a closure condition for the MP trap geometry.

Combining Eq. (9) with Eq. (10) yields

$$
\begin{aligned}
\boldsymbol{\nabla} \cdot\left(\frac{e n v_{\|}}{c B} \mathbf{B}\right)= & \nabla\left[\frac{1}{B^{2}} \frac{\partial p}{\partial \psi}\right] \cdot \nabla \psi \times \nabla \chi \\
& +\nabla\left[\frac{1}{B^{2}} \frac{\partial p}{\partial \vartheta}\right] \cdot \nabla \vartheta \times \nabla \chi
\end{aligned}
$$

The left-hand side is transformed according to

$$
\boldsymbol{\nabla} \cdot\left(\frac{e n v_{\|}}{c B} \mathbf{B}\right)=B^{2} \frac{\partial}{\partial \chi} \frac{e n v_{\|}}{c B} .
$$

Computing the gradients in the right-hand side, one has to take into account that triple products with two gradients of the same function are equal to zero, for example, $\nabla \psi$. $\boldsymbol{\nabla} \psi \times \nabla \chi=0$. Reminding that $B$ is considered here as a function of $\psi, \vartheta$, and $\chi$, and $p$ is a function of $\psi, \vartheta$, and $\phi$, one obtains

$$
\begin{aligned}
B^{2} \frac{\partial}{\partial \chi} \frac{e n v_{\|}}{c B}= & \left(\frac{\partial}{\partial \vartheta} \frac{1}{B^{2}}\right) \frac{\partial p}{\partial \psi} \boldsymbol{\nabla} \cdot \boldsymbol{\nabla} \psi \times \nabla \chi+\left(\frac{\partial}{\partial \psi} \frac{1}{B^{2}}\right) \frac{\partial p}{\partial \vartheta} \boldsymbol{\nabla} \psi \cdot \nabla \vartheta \times \nabla \chi+\frac{1}{B^{2}} \frac{\partial^{2} p}{\partial \vartheta \partial \psi} \boldsymbol{\nabla} \vartheta \cdot \nabla \psi \times \nabla \chi+\frac{1}{B^{2}} \frac{\partial^{2} p}{\partial \psi \partial \vartheta} \nabla \psi \cdot \nabla \vartheta \\
& \times \nabla \chi+\frac{1}{B^{2}} \frac{\partial^{2} p}{\partial \phi \partial \psi} \boldsymbol{\nabla} \phi \cdot \nabla \psi \times \nabla \chi+\frac{1}{B^{2}} \frac{\partial^{2} p}{\partial \phi \partial \vartheta} \boldsymbol{\nabla} \phi \cdot \nabla \vartheta \times \nabla \chi
\end{aligned}
$$

Since $\nabla \psi \cdot \nabla \vartheta \times \nabla \chi=B^{2}$ and $\nabla \vartheta \cdot \nabla \psi \times \nabla \chi=-B^{2}$, the third and fourth terms in the last equation cancel each other. In the fifth term one can change the order of the partial derivatives over $\phi$ and $\psi$ and then make use of the equality $\partial p / \partial \phi=-e p / T$. The triple product $\boldsymbol{\nabla} \phi \cdot \nabla \psi \times \nabla \chi$ is equal to $(\partial \phi / \partial \vartheta) \nabla \vartheta \cdot \nabla \psi \times \nabla \chi=-B^{2}(\partial \phi / \partial \vartheta)$. The sixth term is transformed in a similar way. Dividing both sides of the last equation by $B^{2}$ leads to 


$$
\begin{aligned}
\frac{\partial}{\partial \chi} \frac{e n v_{\|}}{c B}= & -\frac{\partial p}{\partial \psi} \frac{\partial}{\partial \vartheta} \frac{1}{B^{2}}+\frac{\partial p}{\partial \vartheta} \frac{\partial}{\partial \psi} \frac{1}{B^{2}}+\frac{1}{B^{2}} \frac{\partial e \phi}{\partial \vartheta} \frac{\partial}{\partial \psi} \frac{p}{T} \\
& -\frac{1}{B^{2}} \frac{\partial e \phi}{\partial \psi} \frac{\partial}{\partial \vartheta} \frac{p}{T}
\end{aligned}
$$

This equation allows one to calculate the plasma current along the magnetic field lines. Since the current vanishes at the ends of the plasma column, the integral of the righthand side over the entire range of $\chi$ must be equal to zero. This yields the "solvability condition"

$$
\begin{aligned}
0= & \int_{-\infty}^{\infty}\left\{-\frac{\partial p}{\partial \psi} \frac{\partial}{\partial \vartheta} \frac{1}{B^{2}}+\frac{\partial p}{\partial \vartheta} \frac{\partial}{\partial \psi} \frac{1}{B^{2}}+\frac{1}{B^{2}} \frac{\partial e \phi}{\partial \vartheta} \frac{\partial}{\partial \psi} \frac{p}{T}\right. \\
& \left.-\frac{1}{B^{2}} \frac{\partial e \phi}{\partial \psi} \frac{\partial}{\partial \vartheta} \frac{p}{T}\right\} d \chi
\end{aligned}
$$

where the integration is formally extended over an infinite interval (actually it covers the interval of a magnetic field line where the plasma pressure $p$ is nonzero).

The constraint (14) interrelates two functions of $\psi$ and $\vartheta$, namely $N$ and $T$, and, in general, it allows determining $N(\psi, \vartheta)$ if $T(\psi, \vartheta)$ is given or vice versa. One can argue, however, that $T(\psi, \vartheta)$ is not completely independent of $N(\psi, \vartheta)$. Indeed, a differential plasma rotation would result in a fast sharpening of the temperature gradient across the plasma streamlines so that even a weak transverse thermal conductivity effectively flattens the temperature along the streamlines. Therefore one can assume that $\mathbf{v} \cdot \boldsymbol{\nabla} T=0$ in addition to the condition $\mathbf{B} \cdot \boldsymbol{\nabla} T=0$ used in the derivation of Eq. (4). Dotting Eq. (9) with $\nabla T$ one finally concludes that $T$ depends on $\psi$ and $\vartheta$ through the dependence of $N$ on these coordinates, i.e., $T(\psi, \vartheta)=T(N(\psi, \vartheta))$. For the sake of simplicity it is assumed below that $T=$ const. This assumption is relevant to the state of global thermal equilibrium [12,13], which is also characterized by a rigid plasma rotation.

The parallel current constraint (14), together with the Poisson equation (5), allows also computing the plasma currents induced by a magnetic field perturbation in a nonneutral plasma confined in a MP trap. These currents can be thought of as an analog of the Pfirsch-Schlüter currents in Tokamaks [14] or the Stupakov currents in tandem mirrors $[3,15]$. However, they appear even in the case of a uniform magnetic tilt which does not give rise to any magnetic drift, whereas both Pfirsch's-Schlüter's and Stupakov's currents originate from magnetic drifts.

Considering the case of a weak magnetic perturbation, $\epsilon \ll 1$, the unknown functions $\phi$ and $N$ can be sought in the form $\phi(\psi, \vartheta, \chi)=\phi_{0}(\psi, \chi)+\epsilon \phi_{1}(\psi, \vartheta, \chi)$ and $N(\psi, \vartheta)=N_{0}(\psi)+\epsilon N_{1}(\psi, \vartheta)$. The linearized versions of Eqs. (14) and (5) can be readily solved in the region far from the plasma column ends, where the unperturbed electric potential $\phi_{0}=\phi_{0}(\psi)$ does not depend on $\chi$. An example of solution is shown in Fig. 1.

Omitting the details of the calculations, in the case of a uniform magnetic field $B_{*}$ tilted by a small angle $\alpha$ with

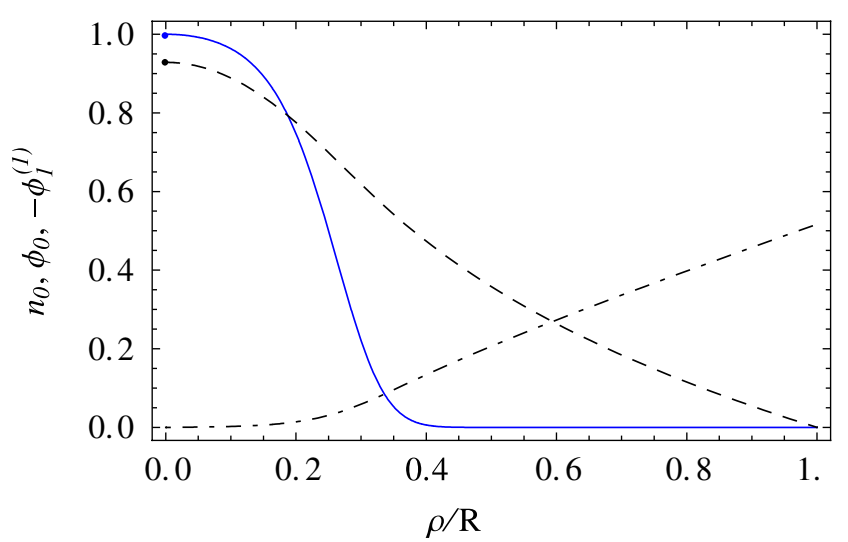

FIG. 1 (color online). Unperturbed density $n_{0}$ (solid line), unperturbed electric potential $\phi_{0}$ (dashed line), and radial part $\phi_{1}^{(1)}$ (dot-dashed line) of the perturbed potential used for calculating the Pfirsch-Schlüter currents vs the flux radius $\rho=$ $\left(2 \psi / B_{*}\right)^{1 / 2}$ normalized over the radius $R$ of the MP trap. The density is normalized by its maximal value $n_{*}$, and the potentials by $(T / e)\left(a / \lambda_{D}\right)^{2}$, with $\lambda_{D} \equiv\left[T /\left(4 \pi e^{2} n_{*}\right)\right]^{1 / 2}$ the Debye length. The density profiles corresponds to a global thermal equilibrium with a column radius $a / R=0.25$ (computed at $1 / 2$ of the maximal density), and $\lambda_{D} / R=0.05$.

respect to the axis of the trap, the perturbed potential can be written as

$$
\phi_{1}(\psi, \vartheta, \chi)=\phi_{1}^{(1)}(\psi)\left(\alpha \chi / B_{*}\right) \cos \vartheta
$$

while $N_{1}=0$ if $\chi=0$ in the midplane of the plasma column (this can be accomplished with a proper choice

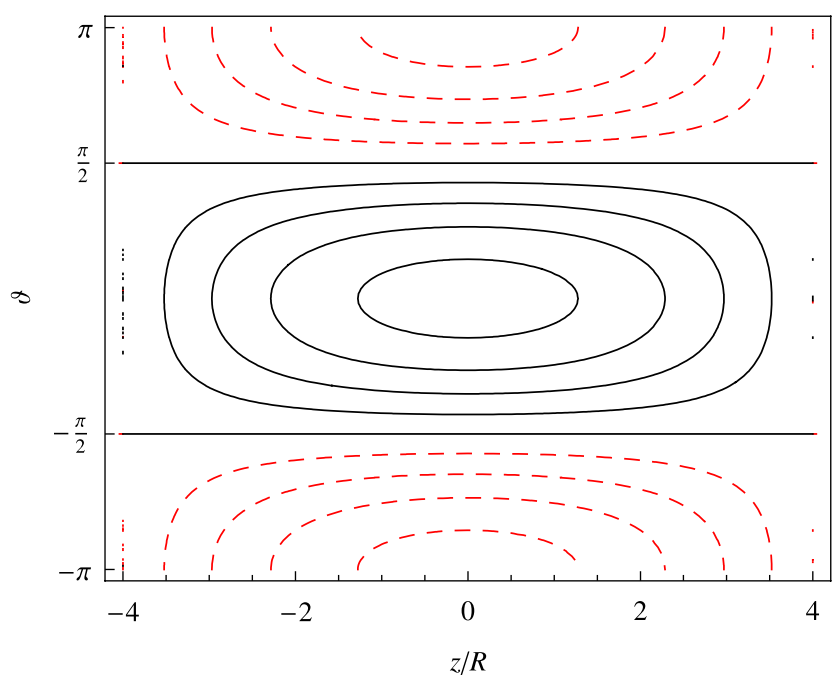

FIG. 2 (color online). Level curves of $\Psi_{1}$ (streamlines of the asymmetry-induced current density) on a flux surface with $\rho / R=0.25$ for a uniform magnetic field with a tilt angle $\alpha=$ $1^{\circ}$. Solid and dashed lines correspond to a clockwise and a counterclockwise flowing current, respectively. The total length of the plasma column is $L=8 R$. Other parameters are indicated in Fig. 1. 


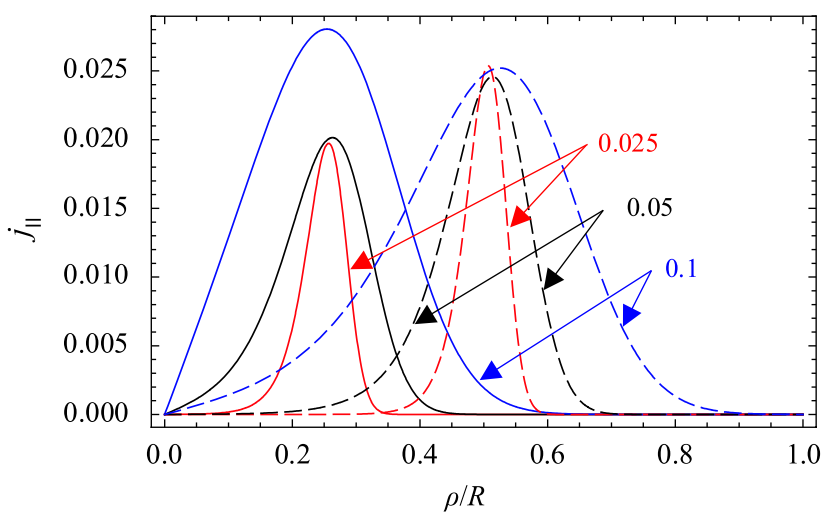

FIG. 3 (color online). Radial profiles of the parallel current density $j_{\|}$for a uniform magnetic tilt along the ray $\vartheta=\pi / 2$, $\chi=0$ (midplane of the trap) for $a / R=0.25$ (solid lines), $a / R=0.5$ (dashed lines) and various values of $\lambda_{D} / R$ (indicated on the plot); $j_{\|}$is normalized by $\left(\alpha c n_{*} T / B_{*} \lambda_{D}\right)(L / R)^{2}\left(a / \lambda_{D}\right)^{2}$.

of the origin of the system of coordinates). The contravariant components of the electric current $\mathbf{j}=e n \mathbf{v}$ determined from Eqs. (9), (11), and (12) assume the following elegant form

$$
j^{i}=\left\{-c \frac{\partial p}{\partial \vartheta}, c \frac{\partial p}{\partial \psi}, e n v_{\|} B\right\}
$$

where

$$
e n v_{\|} B=c \frac{\partial N_{0}}{\partial \psi} \int_{-\infty}^{\chi} \frac{\partial e \phi_{1}}{\partial \vartheta} \exp \left(-\frac{e \phi_{0}}{T}\right) d \chi .
$$

Introducing the flux function

$$
\Psi_{1} \equiv \frac{c T}{B_{*}^{2}} \frac{\partial N_{0}}{\partial \psi} \int_{-\infty}^{\chi} \frac{e \phi_{1}}{T} \exp \left(-\frac{e \phi_{0}}{T}\right) d \chi,
$$

the asymmetry-induced part of the electric current can be cast in the vector form

$$
\mathbf{j}_{1}=\nabla \psi \times \nabla \Psi_{1} .
$$

This equation shows that the radial current density vanishes, $j_{1}^{1}=0$, and that the streamlines of $\mathbf{j}_{1}$ within a given flux surface $\psi=$ const coincide with the contours $\Psi_{1}=$ const. The level curves of $\Psi_{1}$ for a given flux radius $\rho=$ $\left(2 \psi / B_{*}\right)^{1 / 2}$ in the case of a plasma density $N_{0}(\psi)$ corresponding to a global thermal equilibrium $[12,13]$ are drawn in Fig. 2. These level curves show a similar topology at each radius for the parallel current density $j_{\|}$. It can be shown that this feature is no longer valid in the general case of a variable magnetic tilt. This contour plot shows that asymmetry-induced currents are almost parallel to the magnetic field lines on the major part of the plasma column except in the proximity of the column ends where they are shorted out azimuthally. In addition, the radial profiles of $j_{\|}$are peaked near the column edge, $\rho \equiv\left(2 \psi / B_{*}\right)^{1 / 2} \sim a$, as shown in Fig. 3, and the maximum value of $j_{\|}$is roughly evaluated as $\left(c T n_{*} / 32 \lambda_{D} B_{*}\right)\left(a / \lambda_{D}\right)^{2}\left(\alpha L^{2} / R^{2}\right)$, where $n_{*}$ is the peak density.

In this Letter a parallel current constraint has been derived that selects a class of admissible plasma equilibria in the trap in the presence of a nonuniform and a nonaxisymmetric magnetic field. In combination with Poisson's equation this constraint provides a full set of equations for determining self-consistent equilibria of non-neutral plasmas in MP traps.

This work was started during a visit of I. K. to the Department of Physics of the University of Milano thanks to support from the Cariplo Foundation and the Landau Network-Centro Volta. The authors are grateful to Professor R. Pozzoli for useful discussions.

[1] J. H. Malmberg and C. F. Driscoll, Phys. Rev. Lett. 44, 654 (1980).

[2] D. H.E. Dubin and T. M. O’Neil, Phys. Plasmas 5, 1305 (1998).

[3] D. D. Ryutov and G. V. Stupakov, in Reviews of Plasma Physics, edited by B. B. Kadomtsev (Consultants Bureau, New York, 1987), Vol. 13, pp. 93-202.

[4] G. W. Hart, Phys. Fluids B 3, 2987 (1991).

[5] R. Chu, J.S. Wurtele, J. Notte, A.J. Peurrung, and J. Fajans, Phys. Fluids B 5, 2378 (1993).

[6] K. Gomberoff, J. Fajans, A. Friedman, D. Grote, J.-L. Vay, and J. S. Wurtele, Phys. Plasmas 14, 102111 (2007).

[7] T.S. Pedersen and A.H. Boozer, Phys. Rev. Lett. 88, 205002 (2002).

[8] I. Kotelnikov, M. Romé, and A. Kabantsev, Phys. Plasmas 13, 092108 (2006).

[9] L. Brillouin, Phys. Rev. 67, 260 (1945).

[10] I. Kotelnikov, M. Romé, and R. Pozzoli, Phys. Lett. A 372, 1445 (2008).

[11] M. D. Kruskal and R. M. Kulsrud, Phys. Fluids 1, 265 (1958).

[12] T. M. O’Neil, Comments Plasma Phys. Control. Fusion 5, 213 (1980).

[13] I. Kotelnikov, R. Pozzoli, and M. Romé, Phys. Plasmas 7, 4396 (2000).

[14] D. Pfirsch and A. Schluter, Max-Planck-Institut Report No. MPI/PA/7/62, 1962 (unpublished).

[15] G. V. Stupakov, Fiz. Plazmy 5, 871 (1987) [Sov. J. Plasma Phys. 5, 486 (1979)]. 